\title{
Ten years from the Antarctic Sub-millimeter Telescope and Remote Observatory
}

\author{
Christopher L. Martin, on behalf of the AST/RO Team \\ Department of Physics and Astronomy, Oberlin College, Oberlin, OH 44074, USA \\ email: Chris.Martin@oberlin.edu
}

\section{The telescope}

Beginning with the winter season of 1995 and for the next ten years, the Antarctic Sub-mm Telescope and Remote Observatory (AST/RO, Stark et al. 1997; Stark et al. 2001; <http://www.cfa.harvard.edu/ASTRO/>), a $1.7 \mathrm{~m}$ diameter, offset Gregorian telescope located at an altitude of $2847 \mathrm{~m}$ at the Amundsen-Scott South Pole Station collected sub-mm and Terahertz data in the $1.3 \mathrm{~mm}$ to $200 \mu \mathrm{m}$ wavelength bands. From its location just a few hundred meters away from the geophysical South Pole, AST/RO was the first sub-mm telescope to over-winter on the polar plateau, a location uniquely suited to high quality sub-mm observations due its very low humidity, high atmospheric stability and thin troposphere (Chamberlin et al. 1997).

While there are a number of scientists around the world still making use of AST/RO data, the telescope itself was regrettably decommissioned in December 2005. This was due in part to the slow drifting of snow at the South Pole which caused the snow level surrounding the building to slowly rise over time. In Fig. 1, you can compare two photos of the telescope building taken a few years after construction and then again from a similar vantage point in late 2005. In the background can also be seen one of the other grand changes to the landscape during this ten year time-span, the construction of the new Amundsen-Scott South Pole Station to replace the iconic dome.

\section{Observing highlights}

Over the past ten years scientific results using AST/RO data have appeared in nearly fifty peer-reviewed articles, seven Ph.D. theses, and numerous conference proceedings. For the full list see the AST/RO web site. While the full details of all of AST/RO's results are far too numerous to list here, I would like to highlight just a few.

- First detection of $\mathrm{C}_{\mathrm{I}}$ emission in the Magellanic Clouds (Bolatto et al. 2000a)

- First detection of $\mathrm{C}_{\mathrm{I}}$ in absorption (Staguhn et al. 1997)

- Surveys of $\mathrm{C}_{\mathrm{I}}$ and $\mathrm{CO} J=7 \rightarrow 6, \mathrm{CO} J=4 \rightarrow 3$, and $\mathrm{CO} J=2 \rightarrow 1$ emission from - The H II region/molecular cloud complex NGC 6334 (Kim \& Narayanan 2006)

- The inner few degrees of the Milky Way (Martin et al. 2004)

- Nine strips across the Galactic plane (Lane, in prep.)

- The Carina spiral arm region around $\eta$ Carinae (Zhang et al. 2001)

- N 159 / N 160 region in the LMC (Bolatto et al. 2000b)

- Lupus Clouds as part of the Spitzer Legacy Program 'Cores to Disks' (Tothill, in prep.)

- Maps in the [N II] $205 \mu \mathrm{m}$ line of G287.57-0.59 using SPIFI

- Numerous PhD theses

- Staguhn (1996), U. Cologne - Galactic Center

- Ingalls (1999), Boston U. - High Latitude Clouds

- Bolatto (2001), Boston U. - Magellanic Clouds 

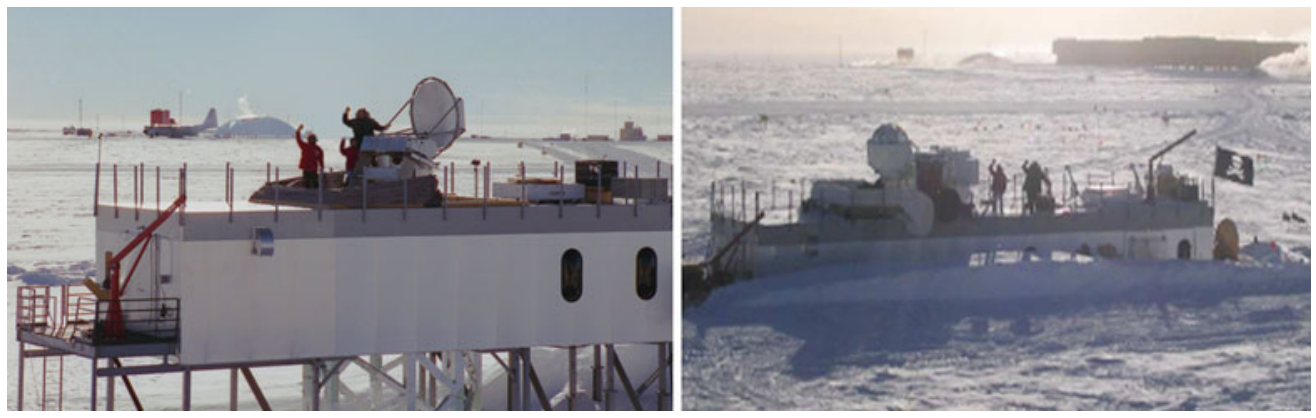

Figure 1. On the left is a picture taken of AST/RO in the late 1990's, a few years after its construction. For contrast, another picture of AST/RO taken in late 2005 during decommissioning is shown on the right. You can clearly see how the snow level has risen around the building over the course of the decade due to drifting in the Dark Sector at the South Pole.

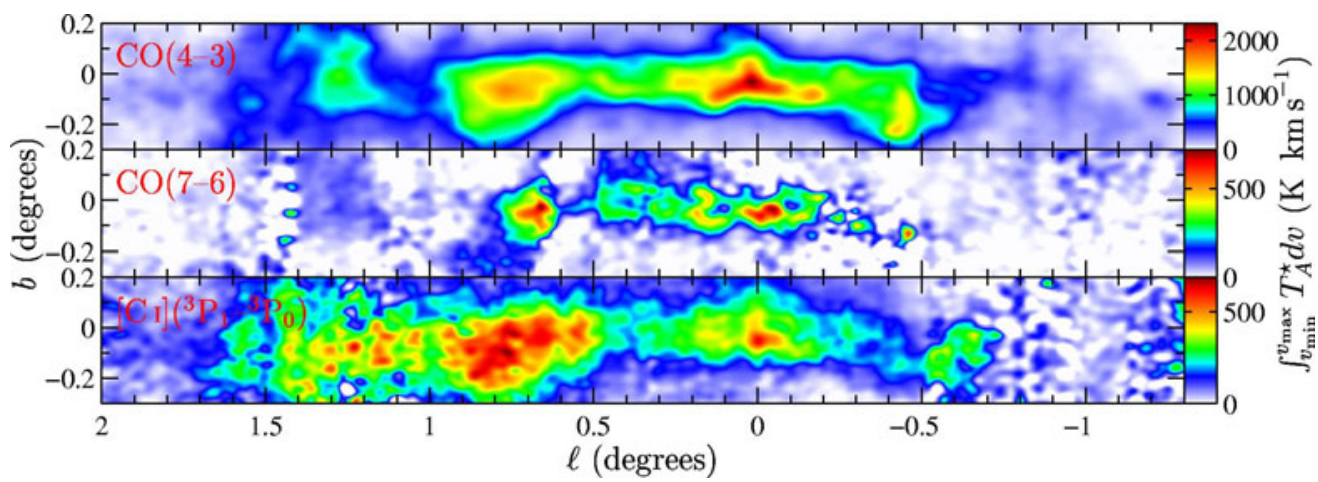

Figure 2. The three panels show spatial-spatial $(\ell, b)$ integrated intensity maps for the three transitions observed with AST/RO in the Galactic Center region. Transitions are identified at left on each panel. The emission is integrated over all velocities where data are available. All have been smoothed to the same $2^{\prime}$ resolution.

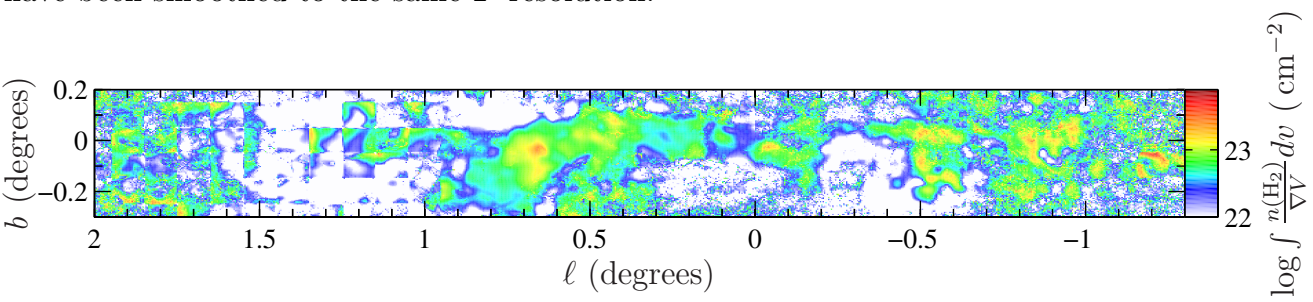

Figure 3. This figure shows one of the most exciting results of AST/RO's Galactic Center Survey. We used a Large Velocity Gradient (LVG) analysis to calculate quantities like the column density (shown here) for every point in our $(\ell, b)$ map. This independent estimate of column density is then available for a wide range of scientific studies. For electronic versions of results from this region as published in Martin et al. (2004), contact the author.

- Huang (2001), Boston U. - Southern H II Regions

- Kulesa (2002), U. Arizona - Dark Interstellar Clouds

○ Groppi (2003), U. Arizona - Star Forming Regions

\section{Galactic Center survey}

While each of the highlights listed above is equally impressive, I would like to focus in on one to serve as an example for what AST/RO has been capable of. 
To understand the strongly excited gas near the center of our own galaxy, detailed surveys in a variety of higher excitation states are required. To aid in this effort, AST/RO completed a fully sampled survey of $\mathrm{CO}(7-6), \mathrm{CO}(4-3),[\mathrm{CI}]\left({ }^{3} \mathrm{P}_{2^{-}}{ }^{3} \mathrm{P}_{1}\right)$, and $[\mathrm{CI}]\left({ }^{3} \mathrm{P}_{1}-{ }^{3} \mathrm{P}_{0}\right)$ in a three square degree region around the Galactic Center (Martin et al. 2004) as shown in Fig. 2. In addition to this inner region, AST/RO has recently completed a survey area around Clump 1 and 2, thus covering the bulk of strongly excited gas near the center of the galaxy.

To collect this dataset required nearly a million distinct telescope pointings over many square degrees of the sky. To handle a sub-mm dataset of this size required the development of new automated observational methodologies, reduction techniques, and visualizations not to mention a substantial amount of observing time. Fortunately AST/RO was designed from the start as a survey instrument with a beam-size of $103-109^{\prime \prime}$ at $461-$ $92 \mathrm{GHz}$ and $58^{\prime \prime}$ at $807 \mathrm{GHz}$. So while covering a few square degrees was still a sizable proposition, we could reasonably contemplate making the multiple passes necessary to acquire data with the uniform signal-to-noise ratio required.

One of the interesting features of this data set is that by using the wide range of emission lines available to AST/RO, we can accurately estimate the kinetic temperature and density over a wide region of the survey using a Large Velocity Gradient (LVG) technique as shown in Fig. 3.

\section{Acknowledgements}

While there are many people to whom thanks is due (see the AST/RO website for the full list), none of the work described here would have been possible without the tireless efforts of the winter-over scientists who spent a full year stationed at the South Pole with the telescope taking the data and maintaining the instrument. AST/RO is supported by NSF grant number ANT-0126090

\section{References}

Bolatto, A. D. 2001, The Interstellar Medium in Low Metallicity Environments, PhD Thesis, Boston University

Bolatto, A. D., Alberto D., Jackson, J. M., Israel, F. P., et al. 2000a, ApJ, 545, 234

Bolatto, A. D., Jackson, J. M., Kraemer, K. E., \& Zhang, X. 2000b, ApJ (Letters), 541, L17

Chamberlin, R. A., Lane, A. P., \& Stark, A. A. 1997, ApJ, 476, 428

Groppi, C. E. 2003, Submillimeter Heterodyne Spectroscopy of Star Forming Regions, PhD Thesis, University of Arizona

Huang, M. 2001, Interstellar Carbon Under the Influence of HII Regions, $\mathrm{PhD}$ Thesis, Boston University

Ingalls, J. G. 1999, Carbon Gas in High Galactic Latitude Molecular Clouds, PhD Thesis, Boston University

Kim, S. and Narayanan, D. 2006, PASJ, 58, 753

Kulesa, C.A. 2002, Molecular Hydrogen and Its Ions in Dark Interstellar Clouds and StarForming Regions, $\mathrm{PhD}$ Thesis, University of Arizona

Lane, A. P. 1998, in: G. Novack \& R. H. Landsberg (eds.), Astrophysics From Antarctica, ASPCS 141,289

Martin, C. L., Walsh, W. M., Xiao, K.-C., et al. 2004, ApJS, 150, 239

Staguhn, J. 1996, Observations Towards the Sgr C Region Near the Center of our Galaxy, PhD Thesis, University of Cologne

Staguhn, J., Stutzki, J., Chamberlin, R. A., et al. 1997, ApJ, 491, 191

Stark, A. A., Bally, J., Balm, S. P., et al. 2001 PASP, 113, 567

Stark, A. A., et al. 1997, Rev. Sci. Instr., 68, 2200

Zhang, X., Lee, Y., Bolatto, A., and Stark, A. A. 2001, ApJ, 553, 274 